\title{
Solution Structures of Spinach Acyl Carrier Protein with Decanoate and Stearate ${ }^{\dagger}$
}

\author{
Gregory A. Zornetzer, Brian G. Fox, and John L. Markley* \\ Department of Biochemistry, University of Wisconsin-Madison, 433 Babcock Drive, Madison, \\ Wisconsin, USA 53706-1549
}

\begin{abstract}
Acyl carrier protein (ACP) is a cofactor in a variety of biosynthetic pathways, including fatty acid metabolism. Thus it is of interest to determine structures of physiologically relevant ACP-fatty acid complexes. We report here the NMR solution structures of spinach ACP with decanoate (10:0-ACP) and stearate (18:0-ACP) attached to the 4 ' phosphopantetheine prosthetic group. The protein in the fatty acid complexes adopts a single conformer, unlike apo- and holo-ACP, which interconvert in solution between two major conformers. The protein component of both 10:0- and 18:0-ACP adopts the four-helix bundle topology characteristic of $\mathrm{ACP}$, and a fatty acid binding cavity was identified in both structures. Portions of the protein close in space to the fatty acid and the 4' phosphopantetheine were identified using filtered/edited NOESY experiments. A docking protocol was used to generate protein structures containing bound fatty acid for 10:0- and 18:0-ACP. In both cases, the predominant structure contained fatty acid bound down the center of the helical bundle, in agreement with the location of the fatty acid binding pockets. These structures demonstrate the conformational flexibility of spinach-ACP and suggest how the protein changes to accommodate its myriad binding partners.
\end{abstract}

Acyl carrier proteins participate in a wide variety of biosynthetic processes, including the synthesis of fatty acids (1), toxins (2), oligosaccharides $(3,4)$, polyketides $(5,6)$, biotin (7), and depsipeptides (8). They ferry small molecules between enzymes involved in biosynthesis by covalent linkage as a thioester to the thiol group of 4'-phosphopantetheine (Figure 1). This prosthetic group is attached by a post-translational modification to a serine (S38 in spinach $\mathrm{ACP}^{1}$ ) in the middle a conserved Asp-Ser-Leu (DSL) sequence. Whereas the sequences of different acyl carrier proteins are divergent, they share a similar four-helix bundle topology and often can be interchanged with full activity in reactions in vitro. This prompts the question: are ACPs simply passive molecules that constantly present their cargo to enzymes, or do protein-protein and protein-ligand interactions affect the utility of ACP as an enzyme substrate?

One well-studied biosynthetic process involving ACP is the desaturation of the fatty acid stearate by the enzyme stearoyl-ACP $\Delta^{9}$-desaturase $(\Delta 9 \mathrm{D})$. This enzyme, in a reaction dependent on $\mathrm{O}_{2}$ and reducing-equivalent, acts on ACP with an attached stearate (18:0-ACP)

\footnotetext{
${ }^{\dagger}$ This work was supported by the National Institutes of Health Grants R01 GM-50853 to B.G.F. and R01 GM-58667 to J.L.M. NMR data were collected at the National Magnetic Resonance Facility at Madison (NMRFAM), which is supported by grants from the NIH Center for Research Resources Biomedical Research Technology Program (P41 RR-02301) and the National Institutes of General Medical Sciences (P41 GM- GM66326) with additional instrumentation purchased with funds from the University of Wisconsin, the NSF Biological Instrumentation Program, the NIH Shared Instrumentation Program, and the U.S. Department of Agriculture. G.A.Z. was a trainee of the NIH Institutional Biotechnology Pre-Doctoral Training Grant T32 GM-08349. Depositions have been made of NMR data (BMRB 6790, 6962) and coordinates (PDB 2FVE, 2FVF, 2AVA, 2FVA) relevant to the structures of 10:0-ACP and 18:0-ACP.

*To whom correspondence should be addressed: Biochemistry Department, 433 Babcock Drive, Madison, WI 53706. Telephone: (608) 263-9349. Fax: (608) 262-3759. E-mail: markley@nmrfam.wisc.edu.

${ }_{1}^{1}$ Abbreviations: ACP, acyl carrier protein; DSS, 2,2-dimethylsilapentane-5-sulfonic acid; HSQC, heteronuclear single-quantum correlation; $\Delta 9 \mathrm{D}, \Delta^{9}$-desaturase; 18:0-ACP, ACP with bound stearate; 10:0-ACP, ACP with bound decanoate.
} 
to yield oleoyl-ACP ( $\left.c i s-\Delta^{9}-18: 1-\mathrm{ACP}\right) . \Delta 9 \mathrm{D}$ has a strong specificity for the 18-carbon fatty acid, and inserts the double bond specifically between carbons 9 and 10 (9). The binding of 18:0-ACP to $\triangle 9 \mathrm{D}$ is a critical step in the catalytic cycle, because it serves to activate the enzyme's diiron cluster for $\mathrm{O}_{2}$ binding (10) and, in chemically-reduced $\Delta 9 \mathrm{D}$, prompts formation of a stable peroxodiferric state for the iron cluster (11). A deep hydrophobic groove in $\triangle 9 \mathrm{D}$ revealed by X-ray crystallography (12) has been suggested as the fatty acid binding site. This hypothesis is supported by studies showing that ACPs with shorter acyl chains bind more weakly to $\triangle 9 \mathrm{D}$ (13). Thus hydrophobic interactions with the fatty acid are a determinant in the affinity of $\triangle 9 \mathrm{D}$ for acyl-ACP. Protein-protein interactions also appear to be involved because it has been shown that the $K_{\mathrm{m}}$ value for the reaction of 18:0-ACP with $\Delta 9 \mathrm{D}$ depends on the identity of the ACP (9) and because high concentrations of holo-ACP are capable of competing with acyl-ACP for the substrate binding site of $\triangle 9 \mathrm{D}$ (13).

Previous investigations of acyl-ACPs have yielded insight into the interactions between ACP and fatty acid chains. Studies of the binding of acyl-ACPs to hydrophobic resins showed that ACP shields the fatty acid from interactions and that this shielding is more effective for acyl chains 10 carbons or shorter (14). In addition, E. coli ACP was found to undergo subtle conformational changes when bound to fatty acids between 6 and 8 carbons long (15). Fluorine NMR was also used to identify protein residues in contact with a specifically fluorinated fatty acid (16). The X-ray crystal structure of E. coli ACP with an attached butyryl group (17) showed that the short-chain fatty acid binds in the hydrophobic core at the center of the four-helix bundle. However, hydrophobic interaction chromatography data suggested that a portion of a longer fatty acid is exposed to solvent (18).

We present here the solution structures of both 10:0- and 18:0-ACP, including the conformation of the bound phosphopantetheine and fatty acid. This work follows up on the earlier observation of large differences in the ${ }^{15} \mathrm{~N}$ HSQC spectra of 10:0- and 18:0-ACP (19) and is a step toward future investigation of ACP- $\triangle 9 \mathrm{D}$ interactions.

\section{EXPERIMENTAL PROCEDURES}

\section{Sample Preparation}

$\left[U_{-}{ }^{15} \mathrm{~N}\right]-$ labeled 10:0- and 18:0-ACP were produced as previously described (19), and NMR samples were prepared at protein concentrations of $2 \mathrm{mM}$. The same expression system, but with $99 \%\left[U_{-}{ }^{13} \mathrm{C}\right]$-glucose (Cambridge Isotope Laboratories, Andover, MA) as the sole carbon source during stirred-vessel fermentation, was used to produce $>98 \%\left[U_{-}{ }^{13} \mathrm{C}, U_{-}{ }^{15} \mathrm{~N}\right]$-labeled ACP. The extent of labeling was determined by mass spectrometry (Mass Spectrometry Facility, University of Wisconsin Biotechnology Center). $9 \mathrm{mg}$ of $\left[U_{-}{ }^{13} \mathrm{C}, U_{-}{ }^{15} \mathrm{~N}\right]$ labeled apoACP was converted to the holo-, 10:0-acyl, and 18:0-acyl forms as previously described (19), (20). These samples were buffer-exchanged into $100 \mathrm{mM} \mathrm{NaCl}$ containing $5 \mathrm{mM}$ MES buffer pH 6.1 and $5 \% \mathrm{D}_{2} \mathrm{O}$ and concentrated to $500 \mu \mathrm{L}$ to yield a sample containing $2 \mathrm{mM}$ acyl-ACP. The sample contained $0.5 \mathrm{mM}$ DSS as a chemical shift reference standard.

Several experiments required a $\left[U_{-}{ }^{13} \mathrm{C}, U_{-}{ }^{15} \mathrm{~N}\right] 18$ :0-ACP sample in pure $\mathrm{D}_{2} \mathrm{O}$. This " $100 \%$ $\mathrm{D}_{2} \mathrm{O}$ " sample was prepared as above with the following additional steps. The sample was frozen in a dry ice/ethanol bath, lyophilized for 1 day, re-dissolved in pure $\mathrm{D}_{2} \mathrm{O}$ (99.996\% deuterium enrichment, Cambridge Isotope Labs, Andover, MA), lyophilized again, and re-dissolved a second time. The sample was then transferred to a dry NMR tube, which was subsequently capped with an LDPE cap (Wilmad Glass, Buena, NJ) and sealed with Parafilm-M (Pechiney Plastic Packaging, Chicago, IL) 


\section{Data Collection for the NMR Structural Studies}

Four NMR spectrometers at the National Magnetic Resonance Facility at Madison were used in collecting data for backbone and sidechain assignments and for NOE constraints: a Bruker DMX $750 \mathrm{MHz}$ spectrometer with a triple axis gradient TXI probe, a Bruker DMX $600 \mathrm{MHz}$ spectrometer with a TXI CryoProbe ${ }^{\mathrm{TM}}$, a Bruker DMX $500 \mathrm{MHz}$ spectrometer with a triple axis gradient TXI probe, and a Varian Inova $600 \mathrm{MHz}$ spectrometer with a ColdProbe ${ }^{\mathrm{TM}}$. The $\left[U-{ }^{15} \mathrm{~N}, U_{-}{ }^{13} \mathrm{C}\right]$-acyl-ACP samples were used for all experiments except where noted. The sample temperature was regulated at $14^{\circ} \mathrm{C}$.

Several multinuclear datasets were collected for backbone and sidechain assignment of both 10:0- and 18:0-ACP: HNCO, HNCACB, C(CO)NH, H(CCO)NH, H(C)CH TOCSY, and TOCSY ${ }^{15}{ }^{15}$ HSQC (using a $\left[U_{-}{ }^{15} \mathrm{~N}\right]$ sample). Data from additional experiments were used in investigating 18:0-ACP: HNCA, CBCA(CO)NH and $\mathrm{HCAN}$ and $\mathrm{HCA}(\mathrm{CO}) \mathrm{N}(21)$ with 18:0$\mathrm{ACP}$ in $100 \% \mathrm{D}_{2} \mathrm{O}$. The latter datasets were used in assigning signals from residues that yielded poor signals in the experiments utilizing ${ }^{1} \mathrm{H}^{\mathrm{N}}$ detection. For distance constraints, two NOESY spectra were collected for each ACP sample: a NOESY $-{ }^{15} \mathrm{~N}$ HSQC (with $\left[U_{-}{ }^{15} \mathrm{~N}\right]$-acyl-ACP samples) and a NOESY- ${ }^{13} \mathrm{C}$ HSQC. In addition, a 3D NOESY- ${ }^{13} \mathrm{C}$ aromatic HSQC was acquired on $\left[U_{-}{ }^{15} \mathrm{~N}, U_{-}{ }^{13} \mathrm{C}\right] 10: 0-\mathrm{ACP}$, and a $2 \mathrm{D}$ NOESY $-{ }^{13} \mathrm{C}$ aromatic HSQC was acquired on 18:0-ACP in $100 \% \mathrm{D}_{2} \mathrm{O}$. All NOESY experiments used a mixing time of $120 \mathrm{~ms}$.

The NMRPipe (22) software suite was used to process the raw datasets. Generally, each dimension was subjected to the following processing steps: linear prediction (when necessary) to estimate missing initial points, cosine window function, zero filling, Fourier transform, phase adjustment, and baseline correction (in some cases). SPARKY 3 software (23) was used to visualize and manually peak-pick spectra for the backbone tracing steps. The NMRDraw program within the NMRPipe distribution was used to automatically peak-pick the NOESY data sets; these peak lists were imported into SPARKY and checked manually. The PISTACHIO automated assignment package (24) was combined with manual assignment steps within the SPARKY software.

\section{Structure Calculation}

The TALOS (25) software was used to derive $\varphi$ and $\Psi$ angular constraints from the backbone assignments. These angular constraints were converted by a PERL script into CYANA aco files, with the limits of the angular constraint set to twice the standard deviation reported by TALOS. The NOESY datasets in Sparky were saved in XEASY peak format and used as input to the NOEassign (26) macro of CYANA 2.0 (27). For 18:0-ACP, hydrogen bond constraints between $\mathrm{H}^{\mathrm{N}}(n)$ and $\mathrm{O}(n-4)$ with a maximum distance of $2.5 \AA$ were then added on the basis of $\alpha$-helices identified in initial structures. These additional constraints were used in a second NOEassign calculation for 18:0-ACP. The 20 best conformers from 100 calculated were saved for both 10:0-ACP and 18:0-ACP.

\section{NMR of the Prosthetic Group}

$\mathrm{A}^{12} \mathrm{C} /{ }^{14} \mathrm{~N}$ gradient-enhanced $z$-filter module was written in the Bruker pulse program language to allow easy modification of traditional NMR experiments with an $f_{1}-12 \mathrm{C} / 14 \mathrm{~N}$ filter. This was based on the design of Ogura et al. (28), with the exception that hard ${ }^{13} \mathrm{C} 180^{\circ}$ pulses were used instead of wide-band inversion pulses and that $90^{\circ}{ }^{13} \mathrm{C}$ pulses were added to generate double-quantum instead of antiphase coherence after the spin echoes. $2 \mathrm{D} f_{1}$-filtered $1 \mathrm{HTOCSY}$ and $1 \mathrm{H}$ NOESY (50 ms mixing time for both spectra) data were collected from the $\left[U_{-}{ }^{13} \mathrm{C}\right.$, $\left.U_{-}{ }^{15} \mathrm{~N}\right]$-acyl-ACP samples. For 18:0-ACP, resonances from atoms in the cofactor neighboring the phosphopantetheine phosphorus were identified using a $1 \mathrm{D}^{1} \mathrm{H}_{-}{ }^{31} \mathrm{P}$ proton-phosphorus correlation experiment (29). $F_{1}$-filtered NOESY- ${ }^{15} \mathrm{~N}$ HSQC spectra were acquired in $2 \mathrm{D}{ }^{1} \mathrm{H}-{ }^{1} \mathrm{H}$ mode to identify the optimal NOESY mixing time for the $3 \mathrm{D}$ spectrum. For 10:0- 
ACP, 3D $f_{1}$-filtered NOESY-15N HSQC and $f_{1}$-filtered NOESY- ${ }^{13} \mathrm{C}$ HSQC spectra were collected with a $180 \mathrm{~ms}$ mixing time. For 18:0-ACP, a $3 \mathrm{D} f_{1}$-filtered NOESY- ${ }^{15} \mathrm{~N}$ HSQC spectrum was acquired with a $300 \mathrm{~ms}$ mixing time.

\section{XPLOR Model Building}

Molecular topology and parameter files were created for 4' phosphopantetheine, stearate, and decanoate using distances, angles, and improper values from protein-1.0.top, protein-1.0.par, nucleic-1.0.top, and nucleic-1.0.par (from XPLOR-NIH 2.12 (30)) where possible. Except for the thioester sulfur and carbonyl carbon, existing atom types were used to describe the prosthetic group atoms. Bond lengths and angles for the thioester carbonyl were derived from neutron diffraction studies of ester-containing compounds CHOLAD04 (31) and ACCHOB12 (32) in the Cambridge Structural Database (Cambridge Crystallographic Data Centre, Cambridge, UK). Bond lengths for the thioester sulfur were derived from TOPPIX01 (33) in the Cambridge Structural Database. Atom names for the 4' phosphopantetheine, stearate and decanoate heavy atoms were taken from the residues PNS, STE, and DKA, respectively, in the HIC-UP small molecule library (34). Protons were numbered according to the attached heavy atom, and an additional designator $(1,2$, or 3$)$ was appended to specify prochiral and methyl protons. The patch feature of XPLOR was used to add prosthetic groups onto the peptide backbone of ACP. For both 10:0-ACP and 18:0-ACP, 20 protein structures from CYANA were loaded into XPLOR-NIH, and the addUnknownAtoms facility was used to generate structures containing an initial random-coil phosphopantetheine and fatty acid attached to the protein. These structures were used as a starting point for the docking protocol.

\section{Experimentally Ambiguous NOESY Constraints}

For docking the fatty acid into the 10:0-ACP structure, data from the filtered/edited NOESY $-{ }^{15} \mathrm{~N}$ and NOESY $-{ }^{13} \mathrm{C}$ HSQC spectra were used. A particular heavy atom $(\mathrm{C}$ or $\mathrm{N})$ of the protein was judged to be "close" to the fatty acid if its attached proton(s) exhibited $2 \mathrm{NOE}$ peaks in the fatty acid region of the NOESY spectrum or involved in a "significant contact" if it exhibited 3 or more NOE peaks in the fatty acid region. For each significant contact, an ambiguous constraint of $4 \AA$ was assigned between the protein heavy atom and any atom in the fatty acid; for each close contact, a $4.5 \AA$ constraint was similarly assigned. A total of 11 contacts for 10:0-ACP were identified (see results), and these provided the constraints to dock the fatty acid into the 10:0-ACP structure.

In the case of the more dynamic 18:0-ACP, the filtered/edited NOESY- $-{ }^{15} \mathrm{~N}$ HSQC results were scored differently. We inferred a contact only if 2 or more NOE peaks were observed between a protein backbone amide proton and signals in the fatty acid region, and for each contact, we introduced an ambiguous $6 \AA$ constraint between the amide nitrogen atom and any fatty acid atom. This resulted in 15 contacts (see results). Four of these contacts were also assigned ambiguously to amide nitrogens due to overlap in the ${ }^{15} \mathrm{~N}$ HSQC.

\section{Fatty Acid Docking}

In a first step of simulated annealing, the protein was held rigid, while the fatty acid and phosphopantetheine were allowed to move freely to satisfy protein-fatty acid NOE constraints. As the simulation cooled, van der Waals constraints were added to force the fatty acid into an acceptable conformation. For 18:0-ACP, residues 31-40 were allowed to move during this first step, but torsion angle database potentials $(35,36,37)$ were used to limit their motion. The final refinement step allowed motion of both the protein and prosthetic group and involved simulated annealing using van der Waals, torsion angle database potentials, ambiguous NOEs, intraprotein NOEs (from CYANA), and dihedral angle constraints. 


\section{Cluster Analysis}

To search for common fatty acid conformations, we used a clustering strategy based on the program HADDOCK (38). All 200 structures were backbone fitted (excluding residues 31-40 in 18:0-ACP). For 10:0-ACP, the rms deviation between the fatty acid heavy atoms was calculated using XPLOR. For 18:0-ACP, the rms deviation between the heavy atoms C15 through C18 was similarly calculated. The program cluster_struc from HADDOCK 1.3 was used to find clusters of fatty acid structures. A $2.0 \AA$ cutoff was used for 10:0-ACP and a 4.0 $\AA ̊$ cutoff was used for 18:0-ACP. The largest resulting cluster for each acyl-ACP was considered significant and is presented.

\section{RESULTS}

\section{Conformational Differences between Holo-ACP and 18:0-ACP}

The ${ }^{15} \mathrm{~N}$ HSQC spectrum of spinach holo-ACP (Figure 2, red) exhibited 166 peaks. This is nearly twice the number of peaks expected from the protein sequence (91). By contrast, the ${ }^{15} \mathrm{~N}$ HSQC spectrum of spinach 10:0-ACP (Figure 2, blue) exhibited 87 peaks, only 4 fewer than the expected number. However, the ${ }^{15} \mathrm{~N}$ HSQC spectrum of spinach 18:0-ACP (Supporting Information 1) exhibited only 73 peaks, 18 fewer than the expected number. As discussed below, these findings are interpreted as indicating (1) that holo-ACP exists in solution in two conformations of about equal population, (2) that this conformational heterogeneity is resolved when holo-ACP is acylated to yield acyl-ACP, and (3) that when the acyl chain is long, as in 18:0-ACP, dynamic effects from the cofactor lead to line broadening that obscures several NMR signals from the protein.

Analysis of a series of ${ }^{15} \mathrm{~N}$ HSQC spectra of acyl-ACP collected as a function of time revealed the appearance of peaks at the positions of those of holo-ACP. The peaks that grew in had ${ }^{1} \mathrm{H}$ linewidths of around $15 \mathrm{~Hz}$ in contrast to the $18-23 \mathrm{~Hz}$ linewidth of the acyl-ACP peaks. This indicates that 18:0-ACP was being converted to holo-ACP through thioester hydrolysis. We have found it possible to minimize this problem by using MES buffer at $\mathrm{pH} 6.1$ and low temperature; nevertheless, thioester hydrolysis limits the useful lifetime of an NMR sample of 18:0-ACP to about one week. By contrast, 10:0-ACP showed little hydrolysis of the acyl group over a period of two months.

\section{Assignment of Protein NMR Signals}

In the case of 10:0-ACP, all backbone resonances were assigned on the basis of HNCACB and $\mathrm{C}(\mathrm{CO}) \mathrm{NH}$ datasets and a comparison of the NOESY $-{ }^{15} \mathrm{~N}$ HSQC and the TOCSY $-{ }^{15} \mathrm{~N}$ HSQC. Data from C(CO)NH, H(CCO)NH, H(C)CH TOCSY, and TOCSY- ${ }^{15} \mathrm{~N}$ HSQC experiments were used to complete the sidechain assignments. The assignment of 18:0-ACP proved more problematic. We assigned the backbone resonances from residues $1-30,44-65$, and $68-82$ on the basis of the HNCACB and $\mathrm{CBCA}(\mathrm{CO}) \mathrm{NH}$ datasets. These were extended to residues 4143,66 , and 67 with information from $\mathrm{HCAN}$ and $\mathrm{HCA}(\mathrm{CO}) \mathrm{N}$ datasets. At this stage, remaining unassigned peaks in the HSQC spectrum included one intense peak and several weak peaks that were significantly broadened in the proton dimension (Supporting Information, Figure 1). These weak peaks had no detected matches in the HNCA or HNCACB data. Some of these backbone peaks corresponded to signals that were assigned in 10:0-ACP (Supporting Information, Figure 2). But even with this information, the low peak intensity prevented the detection and assignment of sidechain resonances. The $\mathrm{H}(\mathrm{CCO}) \mathrm{NH}, \mathrm{C}(\mathrm{CO}) \mathrm{NH}, \mathrm{HCCH}$ TOCSY and TOCSY $-{ }^{15} \mathrm{~N}$ HSQC datasets were used to manually assign peaks to the sidechains of residues $1-30$ and $41-82$. The NOESY $-{ }^{13} \mathrm{C}$ aromatic $\mathrm{HSQC}$ spectra were used to assign residues 31 and 52 . 


\section{0:0-ACP Protein Structure Determination}

A total of 1481 peaks were picked from the NOESY $-{ }^{15} \mathrm{~N}$ HSQC, a total of 1973 peaks were picked from the NOESY $-{ }^{13} \mathrm{C}$ HSQC, and 34 peaks were picked from the NOESY $-{ }^{13} \mathrm{C}$ aromatic HSQC. In addition, $\varphi$ and $\Psi$ constraints were added for 71 residues based on TALOS (25) analysis of backbone chemical shifts. The NOEassign (26) module of CYANA 2.0 (27) yielded a total of 1536 unique distance constraints (see Table 1 for details). 100 conformers based on these constraints were generated, and the 20 conformers with the lowest energy scores were kept. These structures were well-ordered: $93 \%$ of residues were in the most favored region, $7 \%$ in the additionally allowed region, and $0.1 \%$ in the generously allowed region of the Ramachandran plot. The average backbone rms deviation was $0.32 \AA$. Loop regions were wellordered in the model.

\section{8:0-ACP Protein Structure Determination}

A total of 1067 peaks were picked from the NOESY $-{ }^{15}$ N HSQC, and 2712 peaks were picked from the NOESY $-{ }^{13} \mathrm{C}$ HSQC data. The constraints resulting from these data were supplemented with $62 \varphi$ and $\Psi$ angle constraints derived from TALOS (25) analysis of the assigned chemical shifts (Table 1). As noted above, additional constraints representing hydrogen bonds between $\mathrm{H}^{\mathrm{N}}(n)$ and $\mathrm{O}(n-4)$ were added following the initial structure calculations for regions identified as $\alpha$ helix (residues 3-15, 41-51, 58-61, and 67-78). The NOEassign (26) module of CYANA 2.0 (27) yielded a total of 1772 unique distance constraints (see Table 1 for details), and 100 conformers were calculated on the basis of these. Of these, the 20 conformers with the lowest energy scores in CYANA were retained. The only disordered portion of the structure was the poorly-assigned region comprising residues $31-40$. The structured regions of the 20 refined conformers had a backbone rms deviation of $0.48 \AA$.

Both ACPs exhibited a four-helix bundle structure with a distorted square topology (Figures 3 and 4). In tracing the chain, helix 1 (residues 3-17) is followed by an ordered loop region (residues 18-38), and helix 2 (residues 39-52); a short turn precedes the short helix 3 (residues 58-61), and an extended loop leads to helix 4 (residues 67-79). These helices are packed in a right-handed configuration. The main difference between 10:0-ACP (Figure 3) and 18:0-ACP (Figure 4) is that residues 31 through 40 are disordered due to a lack of experimental constraints. Comparison of the structures also revealed subtle differences in helix packing (Supporting Information, Figure 3). Alignment of the secondary structured regions of 10:0- and 18:0-ACP using the program Dalilite (44) yielded a $1.2 \AA \mathrm{rms}$ deviation. As described below, these changes in helix packing explain the larger binding pocket observed for the 18:0 fatty acid than for the 10:0.

\section{Fatty Acid Binding Cavities}

The 20 conformers for 10:0-ACP and 18:0-ACP were each analyzed using the CastP server $(39,40)$ to identify depressions and cavities within the protein structures that could be fatty acid binding sites. An internal cavity was consistently found in both the 10:0- and 18:0-ACP structures. In 10:0-ACP, the cavity has an average volume of $157 \AA^{3}$, and is bordered by residues V9, F31, T41, I44, V45, E48, E49, V56, K60, A61, I64, A70, V73, I74, and L77. In 18:0-ACP, the cavity is larger: an average of $228 \AA^{3}$ in size. The 18:0-ACP cavity is bordered by the same residues as the 10:0-ACP cavity, with the addition of residues I54, N55, D57, and Q62. In one 18:0-ACP conformer, this cavity was exposed to the exterior of the protein. Our hypothesis that this internal cavity is the fatty acid binding site for ACP is confirmed by NOE data as described below. 


\section{Phosphopantetheine and Fatty Acid Binding Sites}

Because the phosphopantetheine and fatty acid were added in vitro after protein production, they were not labeled with ${ }^{15} \mathrm{~N}$ and ${ }^{13} \mathrm{C} .{ }^{14} \mathrm{~N} /{ }^{12} \mathrm{C}$ filtered homonuclear proton experiments, which took advantage of this labeling scheme, permitted the assignment of ${ }^{1} \mathrm{H}$ NMR signals from the phosphopantetheine group as well as the identification of several suspected fatty acid peaks (documented in BMRB depositions 6790 and 6962). The chemical shifts assigned to the phosphopantetheine in 10:0-ACP and 18:0-ACP agree with each other and in general with previous assignments from Streptomyces roseofulvus polyketide synthesis frenolicin holo$\mathrm{ACP}$ (41). One exception is that the protons alpha to the phosphopantetheine thiol in acyl-ACP are shifted downfield by $0.35 \mathrm{ppm}$ from their positions in the holo-ACP; this difference can be explained by the thioester linkage to the fatty acid. A majority of the fatty acid peaks were clustered between 1.1 and $0.7 \mathrm{ppm}$ and were not assignable because of resonance overlaps. However, peaks were assigned to protons of the fatty acid on the basis of similarity to chemical shifts of corresponding protons in free fatty acid.

The $f_{1}$-filter was also added to 3D NOESY-15N HSQC and NOESY-13C HSQC experiments to detect interactions between the phosphopantetheine/fatty acid and the protein backbone. For 10:0-ACP, a 180 ms NOESY mixing time was sufficient to obtain NOE crosspeaks, whereas a $300 \mathrm{~ms}$ NOESY mixing time was required for $18: 0-\mathrm{ACP}$. Although the NOESY- ${ }^{13} \mathrm{C}$ HSQC experiment was successful with 10:0-ACP, it did not yield usable data from 18:0-ACP.

\section{0:0-ACP Fatty Acid Binding Site and Docking}

For 10:0-ACP, a 3D $f_{1}$-filtered NOESY-15N HSQC spectrum identified four amides with significant contacts (interpreted as 4-Å constraints) to the fatty acid: S32, T41, Q62, and I64. The $3 \mathrm{D} f_{1}$-filtered NOESY-13C HSQC spectrum similarly identified four sidechain carbons with significant contacts (interpreted as 4- $\AA$ constraints) to the fatty acid: $\mathrm{F} 31 \mathrm{CB}, \mathrm{I} 44 \mathrm{CB}$, E48 CB, and A70 CB. Three other carbons were identified as "close" (interpreted as 4.5- $\AA$ constraints): I64 CG1, I74 CA, and I74 CG1. None of the signals providing NOEs to the protein could be assigned to individual protons of the fatty acid. Constraints based on these eleven assignments were used to dock the 10-carbon fatty acid into the ACP structure.

The results of the docking calculations are shown in Figure 3. More than $90 \%$ of the 200 calculated 10:0-ACP structures fell into a group with less than a $2.0 \AA$ A fatty acid rms deviation. The fatty acid is nestled in the space between the four helices and principally interacts with residues from helices 2,3 , and 4 . The fatty acids exist in a mostly extended conformation, allowing most of the dihedral angles to be in the energetically favorable anti position. Extensive protein:fatty acid contacts are visible in the models of a single protein conformer with bound fatty acid (Figure $6 \mathrm{~b}$ and $6 \mathrm{c}$ ).

\section{8:0-ACP Fatty Acid Binding Site and Docking}

A 3D $f_{1}$-filtered NOESY-15N HSQC spectrum with a $300 \mathrm{~ms}$ mixing time exhibited weak NOEs between presumed fatty acid signals $\left({ }^{1} \mathrm{H}\right.$ resonances between 0.7 and $\left.1.1 \mathrm{ppm}\right)$ and the backbone amides of 11 residues: I6, L48, E49, I54, D57, A61, Q62, I64, S65, Q69, and V73. As in 10:0-ACP, none of the signals providing NOEs to the protein could be assigned to individual protons of the fatty acid. Several ambiguous constraints were identified from NOEs between the fatty acid and the backbone amides of several residues: A70 or I74, I12 or K81, D40 or V45, and M46 or E75. $6 \AA$ constraints based on these assignments were used to dock the stearate fatty acid into the 18:0-ACP structure.

40\% of the 18:0-ACP docking conformers comprised the largest clustering of structures (Figure 4). Owing to the disordered phosphopantetheine and the lack of constraints on residues 31-40, the carboxyl end of the stearate was free to sample a variety of different conformations. Despite 
this, the fatty acid binding site of 18:0-ACP is similar to that of the much better defined 10:0ACP. The fatty acid binds at the center of the four-helix bundle and has protein contacts with helices 2, 3, and 4 .

\section{DISCUSSION}

\section{Differences between Holo-ACP and 10:0-ACP}

The ${ }^{15} \mathrm{~N}$ HSQC spectra of holo-ACP and 10:0-ACP (Figure 2) exhibited a striking difference in the number of resolved peaks. Spinach holo-ACP has been described previously as interchanging between two conformations (42), and this is confirmed by our results. The holoACP peaks (Figure 2, red) can be separated into two sets. One set of peaks is generally more intense and is clustered in the center of the spectrum; the sharp, undispersed peaks are diagnostic of a dynamically disordered (largely unfolded) protein. The second set of peaks is less intense but more dispersed. On the basis of these results, we conclude that spinach holoACP exists in equilibrium between a folded and unfolded state. The interchange rate for this equilibrium is slow on the NMR timescale - suggestive of an interchange rate slower than 200 $\mathrm{s}^{-1}$.

Covalent addition of the decanoyl group to phosphopantetheine shifts the equilibrium to a folded conformation. A large number of the peaks from 10:0-ACP line up closely with the set of weaker, well-dispersed peaks from holo-ACP (Figure 2, blue and red, respectively). The small differences in chemical shifts can be explained by changes resulting from introduction of the fatty acid.

As a means of comparing the structures of spinach 10:0- and 18:0-ACP with earlier NOESY data from spinach holo-ACP (43), we obtained the NOE-derived distance constraints for spinach holo-ACP (J Prestegard, personal communication). Our results suggest that the structures of holo- and acyl-ACP are comparable, but the data suggest some specific similarities and differences. Constraints between residues 31 and 70 of holo-ACP are satisfied by the acylACP structures. This suggests that the interactions between helix 4 and the loop region between residues 17-31 are similar in holo- and acyl-ACP. However, the acyl-ACP structures were incompatible with the following holo-ACP NOESY constraints: V9-F52, F31-T41, and F31I44. We suggest that these NOESY contacts are disrupted by binding of the fatty acid at the center of the four helix bundle (as in Figure 3). The presence of fatty acid would push helix 2 away from helix 1, abolishing the V9-F52 NOE contact. We suggest that the loop region between F31 and T41 is the entrance to the fatty acid binding pocket, and the F31-T41 and F31-I44 NOESY contacts are disrupted by the formation of this pocket. Data for 10:0-ACP bear this out; however, dynamic effects in 18:0-ACP obscure all constraints in the region between F31 and D40. The F52-I74 NOESY contacts observed in holo-ACP also require comment. Although they are incompatible with the 10:0-ACP structure, they are satisfied in the 18:0-ACP structure. This result suggests that different conformational changes accompany conversion of holo-ACP to 10:0-ACP and 18:0-ACP. This idea is supported by previous work on $E$ coli acyl-ACP with short (2-8 carbon) acyl chains, which suggested that acylation causes a conformational change in this region (15). In addition, our CastP cavity results indicate that the G53-D57 loop region undergoes a conformational change to accommodate fatty acids larger than 10 carbons. In evaluating these apparent differences, it is important to note that the holoACP sample studied by the Prestegard group contained divalent cations added to reduce conformational exchange. Because the acyl-ACPs investigated here did not contain divalent cations, some of the differences could be unrelated to acylation. Nevertheless, the differences in NOE constraints can be rationalized in terms of our model of fatty acid binding down the center of the hydrophobic core of ACP. Acylation shifts the conformational equilibrium between the dynamically disordered and folded forms of holo-ACP toward the folded state, and the conformational changes in this state resulting from acylation are limited. 


\section{Comparison with Other ACP Structures}

The two structures presented here represent the first three-dimensional structures of physiologically relevant long-chain acyl-ACPs. Previous NMR studies of E. coli acyl-ACP (15) were of samples with shorter fatty acid chains (6-8 carbons), and the sole X-ray structure of an acyl-ACP is of E. coli butyryl-ACP (17). Spinach ACP, which has a $39 \%$ sequence identity to $E$. coli $\mathrm{ACP}$, exhibits a similar four-helix bundle topology. Despite differences in the sequences of the acyl carrier proteins with known three-dimensional structures, alignments of their 3-dimensional structures exhibit a high degree of similarity. The program DaliLite (44) was used to align the backbone structures of 10:0- and 18:0-ACP (excluding residues 1740) with structures of ACP from B. subtilis (45), M. tuberculosis (46), Streptomyces roseofulvus polyketide synthetase frenolicin-ACP (41), Streptomyces rimosus polyketide synthetase oxytetracycline-ACP (47), and E. coli ACP in the holo- (48) and butyryl- (17) forms. The Z-scores for pairwise fits of the spinach acyl-ACPs with these family members are shown in Table 2. The different members of the ACP family all have very similar secondary structures, but the packing and length of the helices in each is different, leading to a variety of Z-scores. Not surprisingly, the highest Z-score is found for the fit between the 10:0- and 18:0-ACP structures. This is followed by the fits between E. coli butyryl-ACP and 18:0-ACP and between E. coli butyryl-ACP and 10:0-ACP. It is surprising that the structure of butyryl-ACP, which contains a 4-carbon acyl chain, more closely resembles 18:0-ACP than 10:0-ACP. It is possible that these results simply reflect the difference in primary sequence between $E$. coli and spinach ACP.

The M. tuberculosis, B. subtilis, oxytetracycline-, and frenolicin-ACP structures have intermediate pairwise alignment scores, between 3 and 6, with spinach acyl-ACPs. By contrast, the structural alignment scores of spinach 18:0- and 10:0-ACPs with E. coli holo-ACP are poor: the respective scores of 1.8 and 2.5 are "not significant" and "barely significant". Given the high degree of structural similarity between the E. coli butyryl-ACP and the spinach acylACPs, these results seem inconsistent. However, the E. coli holo-ACP structure is an older NMR structure and was solved from proton NMR data alone, which provide fewer experimental constraints than more recent multinuclear techniques. In addition, the E. coli holo-ACP structure exists in two forms in fast exchange (49). This further complicated structural refinement of the holo-ACP structure and may have limited its accuracy.

\section{Conformation of the 4'-Phosphopantetheine}

No experimental NOESY constraints were observed which could be used to confine the conformation of the phosphopantetheine moiety. We hypothesize that this is a consequence of dynamic disorder of the phosphopantetheine in solution as observed in previous structures of ACP $(41,46,17)$. One argument in support of phosphopantetheine dynamics involves the ${ }^{1} \mathrm{H}$ linewidths. The amide protons of phosphopantetheine in both 10:0- and 18:0-ACP exhibited $13-15 \mathrm{~Hz}$ linewidths in the directly detected ${ }^{1} \mathrm{H}$ dimension. This is sharper than the $>17 \mathrm{~Hz}$ linewidths seen for the rest of the protein. Further evidence comes from the methyl groups of phosphopantetheine. Whereas a representative methyl peak from the protein (I74 MG2, a methyl group on Ile74) had a ${ }^{1} \mathrm{H}$ linewidth of about $20 \mathrm{~Hz}$, the methyl peaks from phosphopantetheine had ${ }^{1} \mathrm{H}$ linewidths of between 5 and $9 \mathrm{~Hz}$. These data indicate that the phosphopantetheine undergoes segmental motions.

A second line of evidence for phosphopantetheine motion comes from the NOE contacts between protons of the protein and protons on the phosphopantetheine seen in 10:0-ACP (but not in 18:0-ACP). These contacts are diagrammed in Figure 5. Carbon and nitrogen atoms (black and blue, respectively) with bound protons in NOE contact with the phosphopantetheine are shown as spheres, and the ensemble of phosphopantetheine conformations is shown as a white surface. All of these contacting atoms are on the same face of ACP as the 
phosphopantetheine. Based on this, we conclude that the phosphopantetheine and fatty acid are correctly localized on the ACP structure. However, the phosphopantetheine contacts are spread across the end of the 10:0-ACP structure (Figure 5a). Notably, many residues in the 3138 loop region, the beginning of helix 2, and the 61-64 loop region are in contact with the phosphopantetheine. These spheres are close in space to the white surface representing the variety of phosphopantetheine conformations. Notably, the white surface does not extend beyond the NOESY contacts. Thus the conformation of the phosphopantetheine appears to be restrained only by the conformation of the covalently attached fatty acid. The phosphopantetheine in the structural model of 18:0-ACP (Figure 5b) has greater apparent freedom of motion compared with the phosphopantetheine in 10:0-ACP (Figure 5a). This is a consequence of the lack of constraints from protein:phosphopantetheine NOEs, which, in turn, may result from the increased dynamics of the longer acyl chain.

\section{Fatty Acid Binding Site}

We have used two independent methods to characterize the fatty acid binding clefts of spinach 10:0- and 18:0-ACP: identification of a binding cavity using CastP $(39,40)$, and NOE-driven ambiguous docking of the fatty acid into the protein structure. In Figure 6, we compare the results of these methods. The green surface represents the binding pocket identified by CastP, while the fatty acid is shown as balls and sticks. For 10:0-ACP (Figure 6b), the fatty acid fits neatly into the pocket. For 18:0-ACP (Figure 6c), the fit is not as clean owing to the relative freedom of motion available to the longer stearate chain. This reflects the previously described exposure of long-chain fatty acids to solvent (18). In spite of this conformational freedom, the end of the fatty acid inserts into the same pocket as seen for the decanoate in 10:0-ACP. A comparison of the pocket residues with the input data for the docking calculation is shown in Table 2. In general, these data agree, although some parts of the protein do not show NOE contacts with the fatty acid. In particular, 10:0-ACP showed no contacts with residues in helix 1. This may be due to overlap in the protein spectra, or it is possible that the fatty acid is not buried far enough to contact V9. There were also no contacts reported to residues near I54. Although several individual NOE peaks were assigned in this region, they did not meet our threshold for defining a contact. Again, this may be the result of spectral overlap. In the case of 18:0-ACP, NOE contacts with F31 were not observed. However, the ${ }^{15} \mathrm{~N}$ HSQC peak for F31 in 18:0-ACP was not identified: it is likely broadened and buried under the peak for I64. In spite of these minor differences, the CastP pocket data and the NOESY data provide complementary evidence that the fatty acid binding pocket is located between the helices in spinach ACP.

Roujeinikova et al. (17) identified several residues forming the acyl chain binding site in $E$. coli butyryl-ACP. These residues are listed in Table 2. Many of these correspond to residues identified by CastP as forming the fatty acid binding site in 10:0-, and 18:0-ACP and are also shown in Table 2 . This is surprising, since one would expect a butyryl moiety to contact fewer residues than a decanoyl moiety. However, careful examination of the crystal structure reveals that the butyryl group does not contact several of the residues identified in the 10:0-, and 18:0ACP pocket and does not fully occupy the binding pocket (Figure 6a). In particular, the methyl end of the butyryl group is nearest residues $28,42,43,46,62$, and 68. According to CastP, residues $7,11,71$, and 72 do not line the fatty acid binding pocket. In contrast to butyryl-ACP, the decanoyl group in 10:0-ACP (Figure 6b) contacts a larger portion of these pocket residues. Although the localization of the fatty acid in 18:0-ACP is more uncertain, it also occupies a larger portion of the pocket than $E$ coli butyryl ACP (Figure 6c). Based on these results, we suggest that, as expected, E. coli and spinach ACP share a common fatty acid binding site down at center of the helical bundle. As the fatty acid attached to ACP becomes longer, conformational changes in ACP enlarge the pocket to accommodate the fatty acid. This is similar to the induced fit binding of substrates in an enzyme active site. 


\section{Fatty Acids with $>10$ Carbons}

Previous work has shown that E. coli ACP is unable to fully protect fatty acids longer than 10 carbons (18). In this work, we identified cavities in 10:0-ACP and 18:0-ACP of $157 \AA^{3}$ and $228 \AA^{3}$, respectively. It is important to note that this difference in cavity size cannot be explained by the lack of structural constraints in 18:0-ACP involving residues 31-40. Except for Phe31, all of the residues in the fatty acid binding pocket of 18:0-ACP are structured. Instead, an alignment of the 10:0- and 18:0-ACP structures (Supporting Information, Figure 3 ) revealed differences in helix packing. The distance between helices 2 and 4 is greater in 18:0- than in 10:0-ACP. A conformational change around helix 3 also enlarges the pocket in this region and is accompanied by changes in the ${ }^{15} \mathrm{~N}$ HSQC peaks for these residues (documented in BMRB entries 6790 and 6962). Thus ACP accommodates fatty acids of various lengths through rearrangement of its four-helix bundle.

The Vega Multipurpose Toolkit (50) was used to determine the volumes of the decanoate and stearate fatty acids based on idealized structures. These volumes were $178 \AA^{3}$ and $305 \AA^{3}$, respectively. Whereas the decanoate fatty acid is similar in size to the cavity in 10:0-ACP (only about $21 \AA^{3}$, or $13 \%$ larger than the cavity), stearate is about $77 \AA^{3}$, or $33 \%$, larger than the cavity in 18:0-ACP. This is another piece of evidence for solvent accessibility of the fatty acid in 18:0-ACP. Others have suggested that, because a long-chain fatty acid is too large to be accommodated within the hydrophobic core of ACP, it will preferentially localize to a second fatty acid binding site within ACP (17). We see no evidence for this from the NOESY data. Instead, we hypothesize that the fatty acid of 18:0-ACP is more dynamic than that of 10:0$\mathrm{ACP}$ as a result of increased solvent accessibility. This results in the poor behavior of residues 31-40 in 18:0-ACP. This hypothesis will be tested by using NMR relaxation to examine the dynamics of both the protein and fatty acid in ACPs with acyl chains of various lengths.

\section{Biological Implications}

ACP is a unique molecule in biology, because it must host a wide variety of fatty acids and fatty acid metabolites from 4 to 18 carbons in length. By comparing the structures and fatty acid localization of $E$. coli butyryl-ACP (17), spinach 10:0-ACP, and spinach 18:0-ACP (Figure 6), it is clear that ACP undergoes small conformational changes in order to accommodate longer fatty acids. The structures of 10:0-ACP and 18:0-ACP presented here confirm the hypothesis that ACP does not fully protect fatty acids greater than 10 carbons in length $(14,18)$. These results have implications for enzymes involved in ACP-dependent reactions, such as $\Delta 9 \mathrm{D}$. Previous studies have shown that the catalytic efficiency of $\Delta 9 \mathrm{D}$ is reduced (9) and the breakdown of the ES complex for $\Delta 9 \mathrm{D}$ is increased (13) as the acyl chain length is shortened. These results were rationalized in terms of a loss of stabilizing hydrophobic interactions between the shorter acyl chain and the hydrophobic channel, leading to an increase in $k_{\text {off }}$ for the ES complex. The present results suggest that a shorter fatty acid is more strongly anchored in the hydrophobic core of $\mathrm{ACP}$ and that formation of the ES would have to overcome these favorable ACP interactions in order to enter the hydrophobic substrate binding channel. By contrast, longer acyl chains have increased solvent exposure and induce greater flexibility in the phosphopantetheine loop, which may be more easily molded during protein-protein interactions to match the shape of the D9D surface and substrate binding channel. These considerations may apply to other systems that require partitioning of the fatty acid between the hydrophobic core of ACP and an enzyme fatty acid binding site. For example, acyl-ACP with $>12$ carbon fatty acids has been shown to be a negative regulator of $E$. coli $\beta$-ketoacylacyl carrier protein synthase III, and this inhibition is increased for longer fatty acids (51). Thus, fatty acid-ACP interactions may contribute to differential stability of the enzyme:acylACP complex, depending on the nature of the bound substituent. 


\section{Supplementary Material}

Refer to Web version on PubMed Central for supplementary material.

\section{Acknowledgements}

We thank Gabriel Cornilescu, Klaas Hallenga, and Marco Tonelli of the National Magnetic Resonance Facility at Madison for assistance in data collection and analysis. We also thank Adam Steinberg for assistance in figure preparation. PyMOL 0.98 (DeLano Scientific, San Carlos, CA) was used in producing the molecular structures in the figures.

\section{References}

1. Magnuson K, Jackowski S, Rock CO, Cronan JE Jr. Regulation of fatty acid biosynthesis in Escherichia coli. Microbiol Rev 1993;57:522-542. [PubMed: 8246839]

2. Issartel J, Koronakis V, Hughes C. Activation of Escherichia-coli Prohaemolysin to the Mature Toxin by Acyl Carrier Protein-Dependent Fatty Acylation. Nature 1991;351:759-761. [PubMed: 2062368]

3. Rumley M, Therisod H, Weissborn A, Kennedy E. Mechanisms of Regulation of the Biosynthesis of Membrane-Derived Oligosaccharides in Escherichia coli. J Biol Chem 1992;267:11806-11810. [PubMed: 1534803]

4. Therisod H, Weissborn A, Kennedy E. An Essential Function for Acyl Carrier Protein in the Biosynthesis of Membrane-Derived Oligosaccharides of Escherichia coli. Proc Natl Acad Sci 1986;83:7236-7240. [PubMed: 2945202]

5. Shen B, Summers RG, Gramajo H, Bibb MJ, Hutchinson CR. Purification and characterization of the acyl carrier protein of the Streptomyces glaucescens tetracenomycin C polyketide synthase. J Bacteriol 1992;174:3818-3821. [PubMed: 1592832]

6. Summers RG, Ali A, Shen B, Wessel WA, Hutchinson CR. Malonyl-coenzyme A:acyl carrier protein acyltransferase of Streptomyces glaucescens: a possible link between fatty acid and polyketide biosynthesis. Biochemistry 1995;34:9389-9402. [PubMed: 7626609]

7. Sanyal I, Lee S, Flint D. Biosynthesis of pimeloyl-CoA, a biotin precursor in Escherichia coli, follows a modified fatty-acid synthesis pathway - ${ }^{13}$ C-labelling studies. J Am Chem Soc 1994;116:2637-2638.

8. Rusnak F, Sakaitani M, Drueckhammer D, Reichert J, Walsh CT. Biosynthesis of the Escherichia coli siderophore enterobactin: sequence of the entF gene, expression and purification of EntF, and analysis of covalent phosphopantetheine. Biochemistry 1991;30:2916-2927. [PubMed: 1826089]

9. Haas JA, Fox BG. Role of hydrophobic partitioning in substrate selectivity and turnover of the Ricinus communis stearoyl acyl carrier protein $\Delta^{9}$ desaturase. Biochemistry 1999;38:12833-12840. [PubMed: 10504253]

10. Yang Y, Broadwater John A, Pulver SC, Fox BG, Solomon EI. Circular dichroism and magnetic circular dichroism studies of the reduced binuclear non-heme iron site of stearoyl-ACP $\Delta^{9}$ Desaturase: Substrate binding and comparison to ribonucleotide reductase. J Am Chem Soc 1999;121:2770-2783.

11. Broadwater JA, Ai J, Loehr TM, Sanders-Loehr J, Fox BG. Peroxodiferric intermediate of stearoylacyl carrier protein $\Delta^{9}$ desaturase: oxidase reactivity during single turnover and implications for the mechanism of desaturation. Biochemistry 1998;37:14664-14671. [PubMed: 9778341]

12. Lindqvist Y, Huang W, Schneider G, Shanklin J. Crystal structure of $\Delta^{9}$ stearoyl-acyl carrier protein desaturase from castor seed and its relationship to other di-iron proteins. EMBO J 1996;15:40814092. [PubMed: 8861937]

13. Haas JA, Fox BG. Fluorescence anisotropy studies of enzyme-substrate complex formation in stearoyl-ACP desaturase. Biochemistry 2002;41:14472-14481. [PubMed: 12463745]

14. Cronan JE Jr. Molecular properties of short chain acyl thioesters of acyl carrier protein. J Biol Chem 1982;257:5013-5017. [PubMed: 7040391]

15. Mayo KH, Prestegard JH. Acyl carrier protein from Escherichia coli. Structural characterization of short-chain acylated acyl carrier proteins by NMR. Biochemistry 1985;24:7834-7838. [PubMed: 3912008] 
16. Jones PJ, Cioffi EA, Prestegard JH. $\left\{{ }^{19} \mathrm{~F}\right\}-{ }^{1} \mathrm{H}$ heteronuclear nuclear Overhauser effect studies of the acyl chain-binding site of acyl carrier protein. J Biol Chem 1987;262:8963-8965. [PubMed: 3298246]

17. Roujeinikova A, Baldock C, Simon WJ, Gilroy J, Baker PJ, Stuitje AR, Rice DW, Slabas AR, Rafferty JB. X-ray crystallographic studies on butyryl-ACP reveal flexibility of the structure around a putative acyl chain binding site. Structure (Camb) 2002;10:825-835. [PubMed: 12057197]

18. Rock CO, Garwin JL. Preparative enzymatic synthesis and hydrophobic chromatography of acyl-acyl carrier protein. J Biol Chem 1979;254:7123-7128. [PubMed: 379000]

19. Zornetzer GA, White RD, Markley JL, Fox BG. Preparation of isotopically labeled spinach acyl-acyl carrier protein for NMR structural studies. Protein Expr Purif. 2005In press

20. Broadwater JA, Fox BG. Spinach holo-acyl carrier protein: overproduction and phosphopantetheinylation in Escherichia coli BL21(DE3), in vitro acylation, and enzymatic desaturation of histidine-tagged isoform I. Protein Expr Purif 1999;15:314-326. [PubMed: 10092491]

21. Ottiger M, Bax A. An empirical correlation between amide deuterium isotope effects on ${ }^{13} C_{\alpha}$ chemical shifts and protein backbone conformation. J Am Chem Soc 1997;119:8070-8075.

22. Delaglio F, Grzesiek S, Vuister GW, Zhu G, Pfeifer J, Bax A. NMRPipe: a multidimensional spectral processing system based on UNIX pipes. J Biomol NMR 1995;6:277-293. [PubMed: 8520220]

23. Goddard, TD.; Kneller, DG. Sparky 3. University of California; San Francisco:

24. Eghbalnia HR, Bahrami A, Wang L, Assadi A, Markley JL. Probabilistic Identification of Spin Systems and their Assignments including Coil-Helix Inference as Output (PISTACHIO). J Biomol NMR 2005;32:219-233. [PubMed: 16132822]

25. Cornilescu G, Delaglio F, Bax A. Protein backbone angle constraints from searching a database for chemical shift and sequence homology. J Biomol NMR 1999;13:289-302. [PubMed: 10212987]

26. Herrmann T, Guntert P, Wuthrich K. Protein NMR structure determination with automated NOE assignment using the new software CANDID and the torsion angle dynamics algorithm DYANA. J Mol Biol 2002;319:209-227. [PubMed: 12051947]

27. Guntert P, Mumenthaler C, Wuthrich K. Torsion angle dynamics for NMR structure calculation with the new program DYANA. J Mol Biol 1997;273:283-298. [PubMed: 9367762]

28. Ogura K, Terasawa H, Inagaki F. An improved doubled-tuned and isotope-filtered pulse scheme based on a pulsed field gradient and a wide-band inversion shaped pulse. J Biomol NMR 1996;8:492498.

29. Luy B, Marino JP. (1)H-(31)P CPMG-correlated experiments for the assignment of nucleic acids. J Am Chem Soc 2001;123:11306-11307. [PubMed: 11697980]

30. Schwieters CD, Kuszewski JJ, Tjandra N, Clore GM. The Xplor-NIH NMR molecular structure determination package. J Magn Reson 2003;160:65-73. [PubMed: 12565051]

31. Weber HP, Craven BM, Sawzik P, McMullan RK. Crystal structure and thermal vibrations of cholesteryl acetate from neutron diffraction at 123 and 20 K. Acta Crystallogr B 1991;47:116-127. [PubMed: 2025409]

32. Shankland N, Florence AJ, Wilson CC. Single-crystal neutron diffraction analysis of anion-cation interactions in perdeuteroacetylcholine bromide at 100 K. Acta Crystallogr B 1997;53:176-180.

33. Adams RD, Huang M, Huang W. Catalytic ring opening of beta-propiothiolactones by dirhenium and dimanganese carbonyl complexes. Organometallics 1997;16:4479-4485.

34. Kleywegt GJ, Jones TA. Databases in protein crystallography. Acta Crystallogr D Biol Crystallogr 1998;54:1119-1131. [PubMed: 10089488]

35. Kuszewski J, Gronenborn AM, Clore GM. Improving the quality of NMR and crystallographic protein structures by means of a conformational database potential derived from structure databases. Protein Sci 1996;5:1067-1080. [PubMed: 8762138]

36. Kuszewski J, Gronenborn AM, Clore GM. Improvements and extensions in the conformational database potential for the refinement of NMR and X-ray structures of proteins and nucleic acids. $\mathrm{J}$ Magn Reson 1997;125:171-177. [PubMed: 9245376]

37. Kuszewski J, Clore GM. Sources of and solutions to problems in the refinement of protein NMR structures against torsion angle potentials of mean force. J Magn Reson 2000;146:249-254. [PubMed: 11001840] 
38. Dominguez C, Boelens R, Bonvin AMJJ. HADDOCK: a protein-protein docking approach based on biochemical or biophysical information. J Am Chem Soc 2003;125:1731-1737. [PubMed: 12580598]

39. Liang J, Edelsbrunner H, Woodward C. Anatomy of protein pockets and cavities: measurement of binding site geometry and implications for ligand design. Protein Sci 1998;7:1884-1897. [PubMed: 9761470]

40. Binkowski TA, Naghibzadeh S, Liang J. CASTp: Computed Atlas of Surface Topography of proteins. Nucleic Acids Res 2003;31:3352-3355. [PubMed: 12824325]

41. Li Q, Khosla C, Puglisi JD, Liu CW. Solution structure and backbone dynamics of the holo form of the frenolicin acyl carrier protein. Biochemistry 2003;42:4648-4657. [PubMed: 12705828]

42. Kim Y, Prestegard JH. Demonstration of a conformational equilibrium in acyl carrier protein from spinach using rotating frame nuclear magnetic resonance spectroscopy. J Am Chem Soc 1990;112:3707-3709.

43. Oswood MC, Kim Y, Ohlrogge JB, Prestegard JH. Structural homology of spinach acyl carrier protein and Escherichia coli acyl carrier protein based on NMR data. Proteins 1997;27:131-143. [PubMed: 9037718]

44. Holm L, Park J. DaliLite workbench for protein structure comparison. Bioinformatics 2000;16:566567. [PubMed: 10980157]

45. Xu GY, Tam A, Lin L, Hixon J, Fritz CC, Powers R. Solution structure of B. subtilis acyl carrier protein. Structure (Camb) 2001;9:277-287. [PubMed: 11525165]

46. Wong HC, Liu G, Zhang Y, Rock CO, Zheng J. The solution structure of acyl carrier protein from Mycobacterium tuberculosis. J Biol Chem 2002;277:15874-15880. [PubMed: 11825906]

47. Findlow SC, Winsor C, Simpson TJ, Crosby J, Crump MP. Solution structure and dynamics of oxytetracycline polyketide synthase acyl carrier protein from Streptomyces rimosus. Biochemistry 2003;42:8423-8433. [PubMed: 12859187]

48. Kim Y, Prestegard JH. Refinement of the NMR structures for acyl carrier protein with scalar coupling data. Proteins 1990;8:377-385. [PubMed: 2091027]

49. Kim Y, Prestegard JH. A dynamic model for the structure of acyl carrier protein in solution. Biochemistry 1989;28:8792-8797. [PubMed: 2690950]

50. Pedretti A, Villa L, Vistoli G. VEGA: a versatile program to convert, handle and visualize molecular structure on Windows-based PCs. J Mol Graph Model 2002;21:47-49. [PubMed: 12413030]

51. Heath RJ, Rock CO. Inhibition of $\beta$-ketoacyl-acyl carrier protein synthase III (FabH) by acyl-acyl carrier protein in Escherichia coli. J Biol Chem 1996;271:10996-11000. [PubMed: 8631920] 


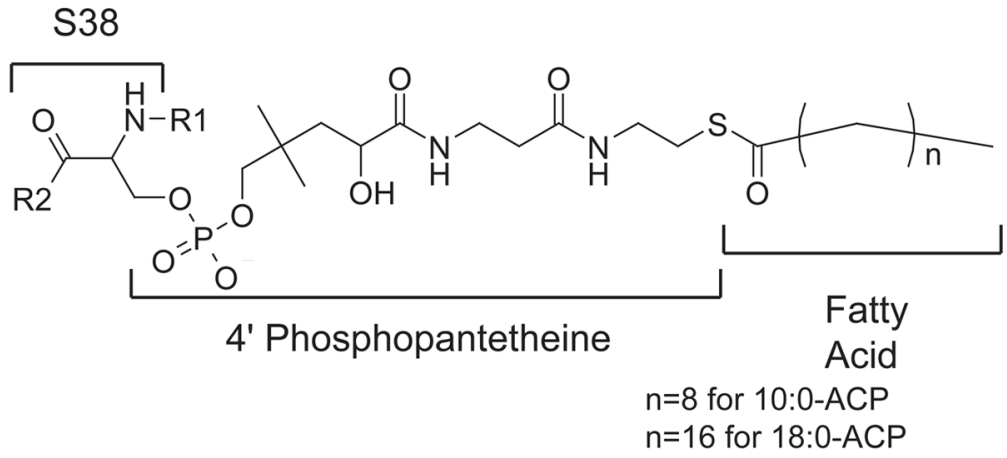

Figure 1.

Covalent structure of the acyl phosphopantetheine modification of Ser38 in spinach ACP. 


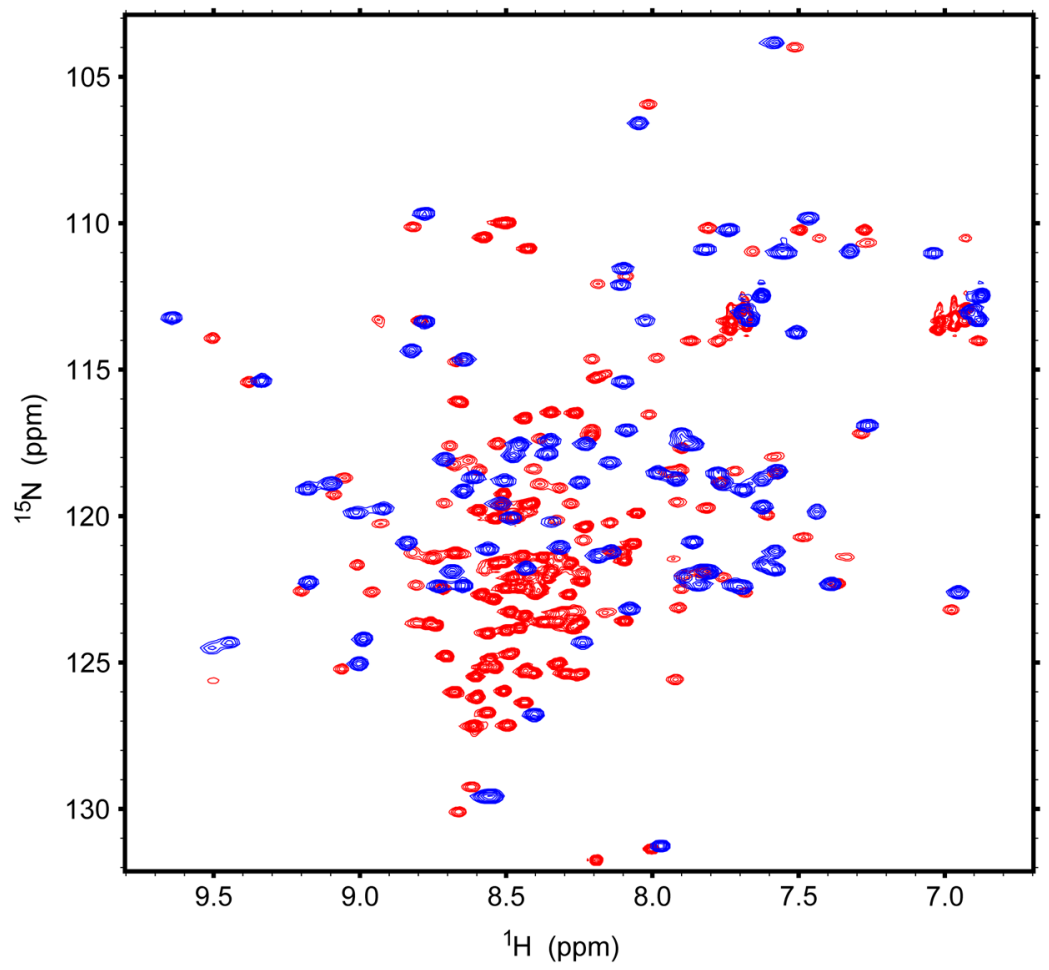

Figure 2.

${ }^{15}$ N HSQC spectra of holo- and 10:0-ACP. Holo-ACP is shown in red, and 10:0-ACP is shown in blue. Samples contained $2 \mathrm{mM}$ protein in $100 \mathrm{mM} \mathrm{NaCl}$, and $10 \mathrm{mM} \mathrm{MES}$. The $\mathrm{pH}$ was 6.1 and the sample temperature was $14^{\circ} \mathrm{C}$. Data were collected on a Bruker DMX $500 \mathrm{MHz}$ spectrometer. 

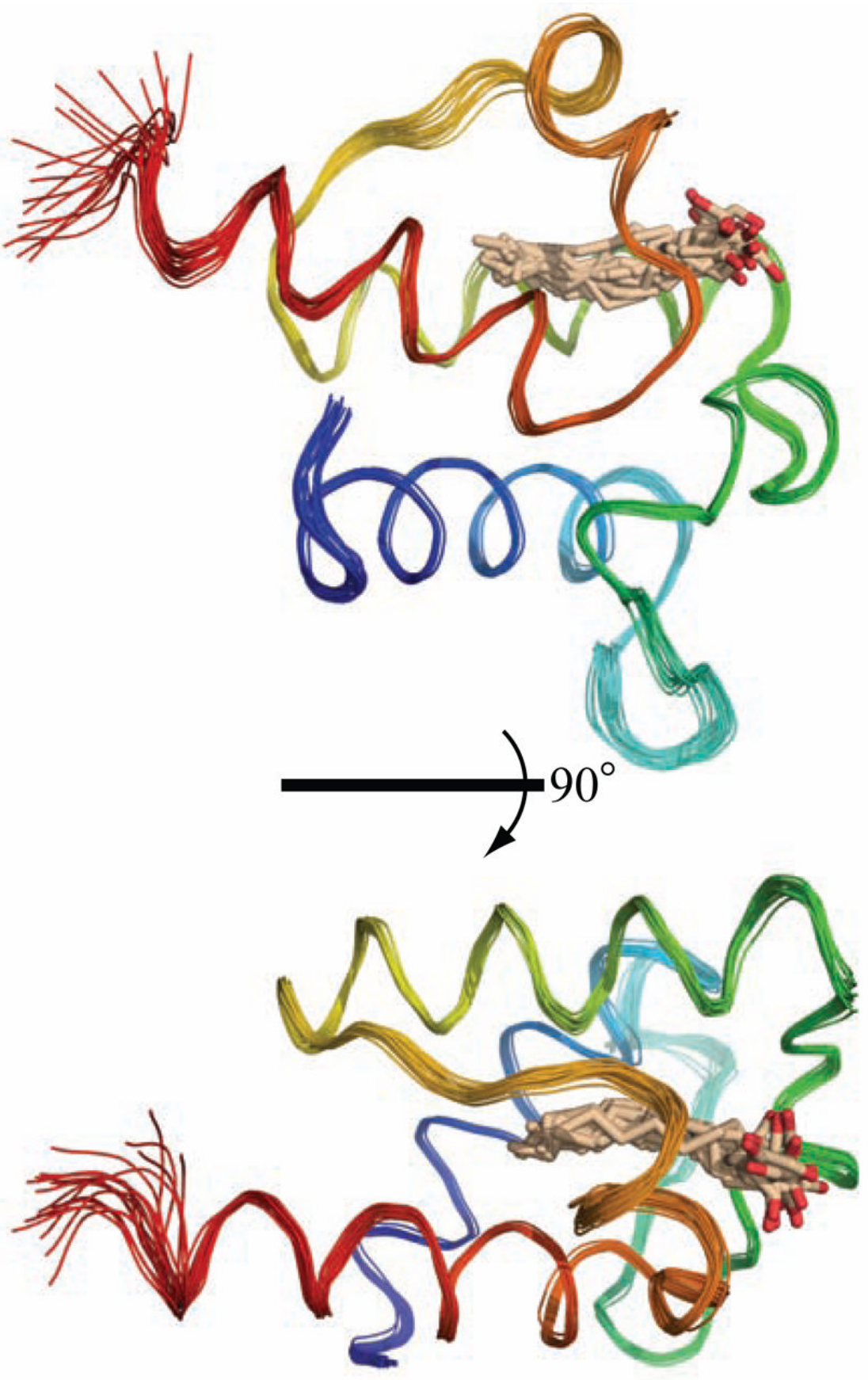

Figure 3.

Two orientations of the ensemble of 20 conformers representing the structure of 10:0-ACP with docked decanoate. The protein backbone $\mathrm{C}^{\alpha}$ trace is shown in rainbow colors (blue Nterminus, red C-terminus). The ensemble of docked fatty acids is shown as beige sticks. 

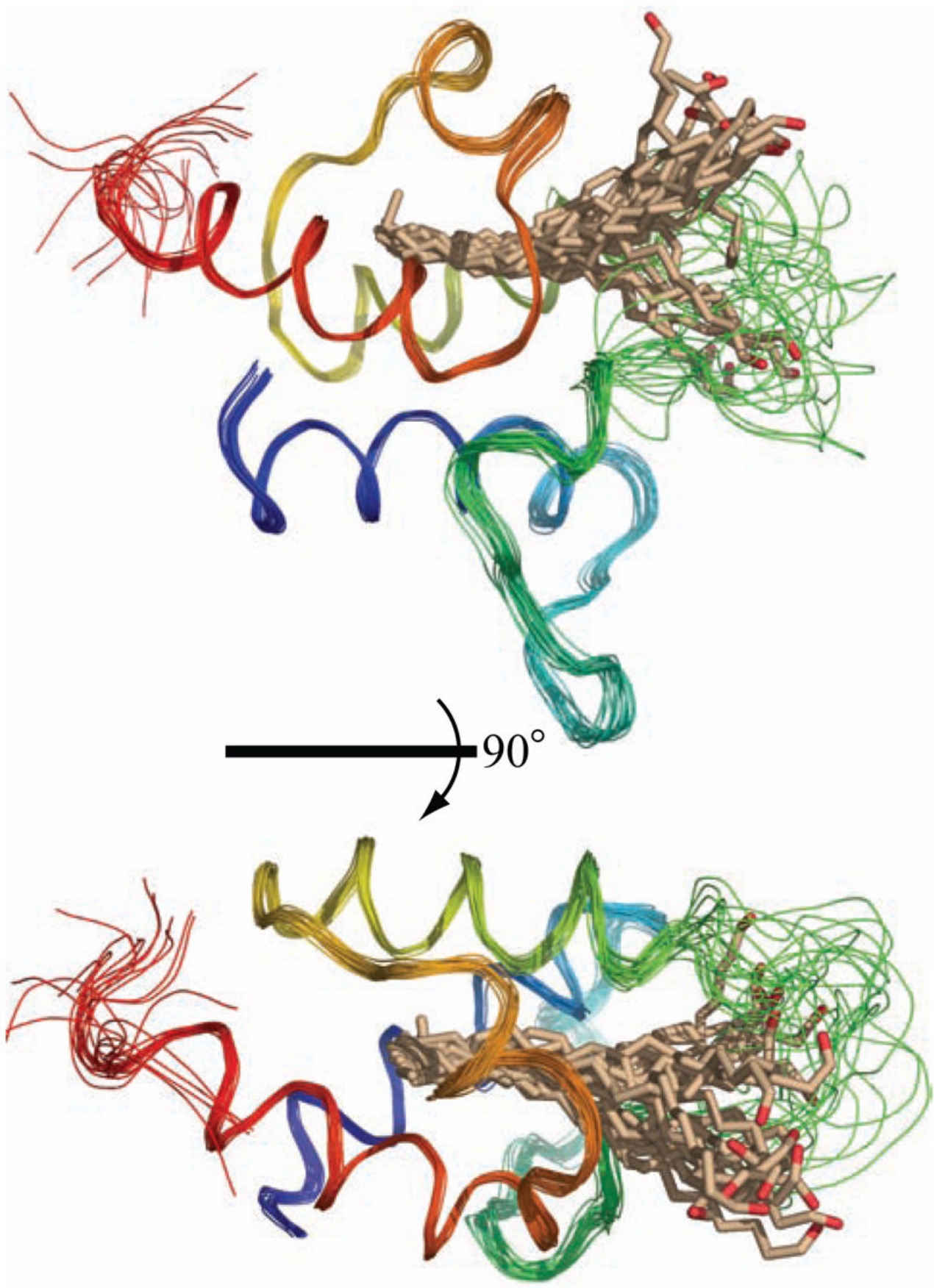

Figure 4.

Two orientations of the ensemble of 20 conformers representing the structure of 18:0-ACP with docked stearate. The protein backbone $\mathrm{C}^{\alpha}$ trace is shown in rainbow colors (blue Nterminus, red C-terminus). The ensemble of docked stearate is shown in beige sticks. 


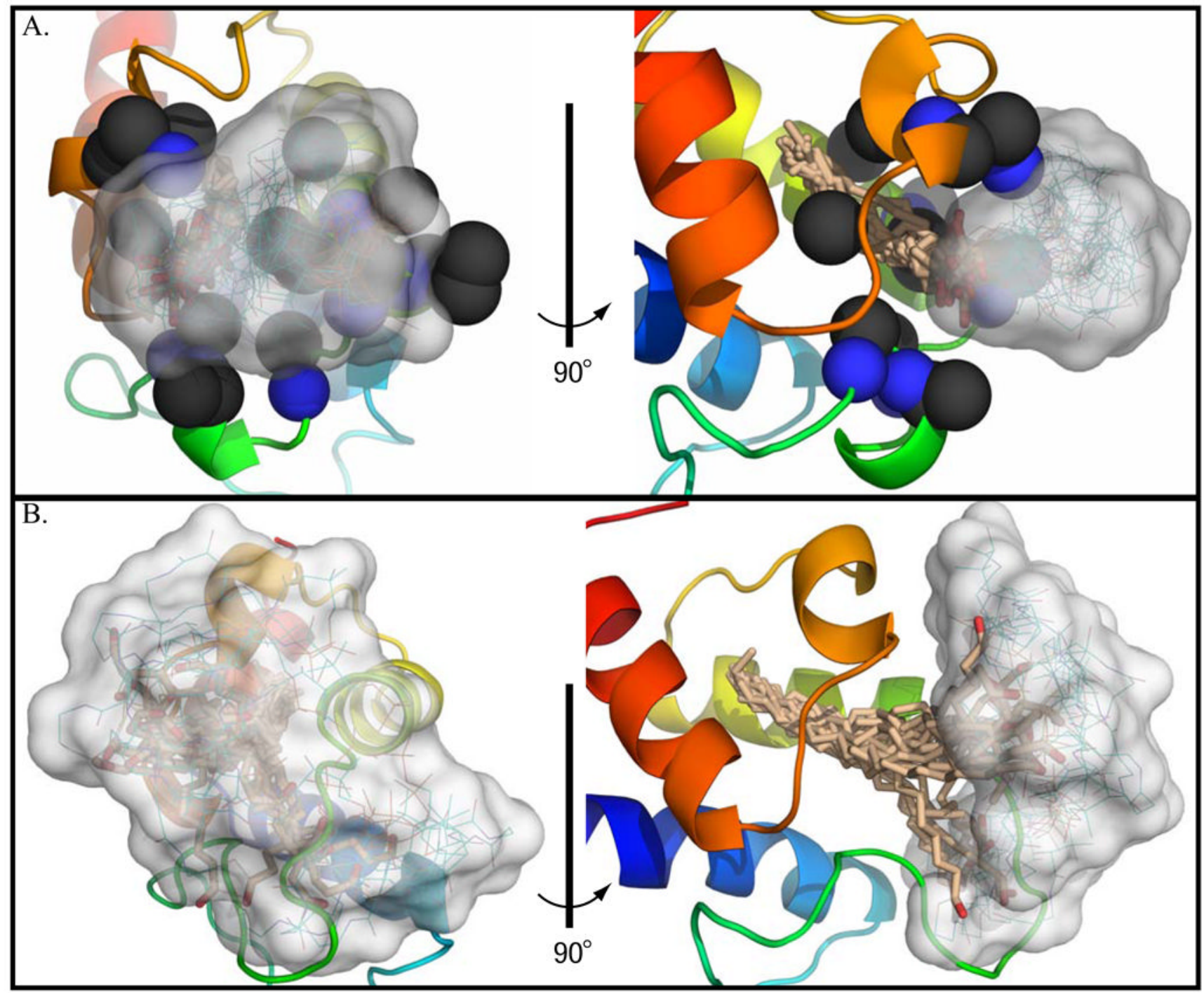

Figure 5.

Two orientations showing the phosphopantetheine localization in 10:0-ACP (a) and 18:0-ACP (b). The ensemble of phosphopantetheine structures is shown as a white surface. The protein is shown as a rainbow ribbon (blue N-terminus, red C-terminus) Carbon and nitrogen (black and blue, respectively) atoms of the protein exhibiting NOE contacts to the phosphopantetheine in 10:0-ACP are shown as spheres. 


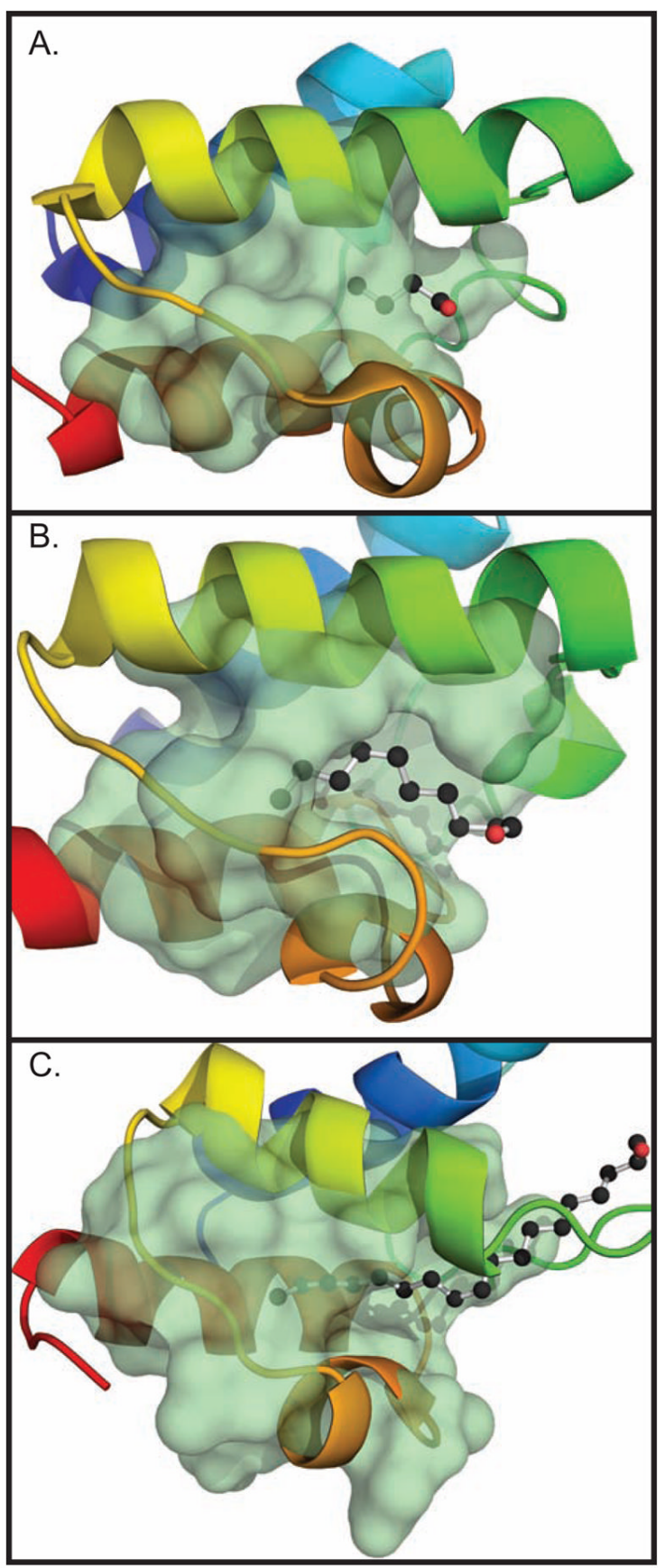

Figure 6.

Fatty acid binding pocket. Residues identified by CastP $(39,40)$ as lining the fatty acid binding pocket are shown as a green surface. The position of the fatty acid is shown as a beige surface enclosing a ball-and-stick representation of the fatty acid. The protein is shown as a rainbow ribbon (blue N-terminus, red C-terminus). (a) The crystal structure of $E$. coli butyryl-ACP (17), (b) one structure of 10:0-ACP, and (c) one structure of 18:0-ACP. 
Table 1

CYANA Structure Statistics for Acyl-ACP Structures.

\begin{tabular}{|c|c|c|}
\hline Acyl Chain & 18:0 & 10:0 \\
\hline \multicolumn{3}{|l|}{ NOE distance constraints } \\
\hline $\begin{array}{l}\text { Intraresidue } \\
\text { Short range } \\
\text { Medium range } \\
\text { Long Range } \\
\text { Additional Constraints }\end{array}$ & $\begin{array}{l}363 \\
445 \\
485 \\
479\end{array}$ & $\begin{array}{l}388 \\
397 \\
436 \\
315\end{array}$ \\
\hline $\begin{array}{l}\text { Hydrogen Bond } \\
\varphi / \Psi \text { Angles } \\
\text { Quality of Structures }\end{array}$ & $\begin{array}{l}20 \\
62\end{array}$ & $\begin{array}{c}0 \\
71\end{array}$ \\
\hline $\begin{array}{l}\text { Residues surveyed } \\
\text { Average rms deviation from the mean }\end{array}$ & $4-28,45-78$ & $4-78$ \\
\hline $\begin{array}{l}\text { Backbone atoms } \\
\text { Heavy atoms } \\
\text { Ramachandran Statistics }\end{array}$ & $\begin{array}{l}0.48 \AA \\
0.96 \AA\end{array}$ & $\begin{array}{l}0.32 \AA \\
0.71 \AA\end{array}$ \\
\hline $\begin{array}{l}\text { Most favored } \\
\text { Additionally allow } \\
\text { Generously allow } \\
\text { Disallowed } \\
\text { Constraint Violations (rms) }\end{array}$ & $\begin{array}{c}86 \% \\
13 \% \\
1 \% \\
0 \%\end{array}$ & $\begin{array}{c}93 \% \\
7 \% \\
0.1 \% \\
0 \%\end{array}$ \\
\hline $\begin{array}{l}\text { NOE distance } \\
\text { Angles }\end{array}$ & $\begin{array}{c}0.0198 \AA \\
0.665^{\circ}\end{array}$ & $\begin{array}{c}0.0023 \AA \\
0.141^{\circ}\end{array}$ \\
\hline
\end{tabular}


Table 2

DaliLite (44) pairwise Z-scores for Structures in the ACP Family.

\begin{tabular}{lllll}
\hline \multirow{2}{*}{ Spinach 18:0-ACP } & PDB & Ref & Spinach 10:0-ACP & Spinach 18:0-ACP \\
\cline { 2 - 5 } & & This work & $\mathbf{1 0 . 2}$ & 7.0 \\
\hline E. coli butyryl-ACP & $1 \mathrm{LOI}$ & $(17)$ & 6.4 & 5.6 \\
\hline Streptomyces rimosus oxytetracycline-ACP & $1 \mathrm{NQ} 4$ & $(47)$ & 5.8 & 4.9 \\
\hline B. subtilis ACP & $1 \mathrm{HY} 8$ & $(45)$ & 5.5 & 4.5 \\
\hline Streptomyces roseofulvus frenolicin-ACP & $1 \mathrm{OR} 5$ & $(41)$ & 3.2 & 3.5 \\
\hline M. tuberculosis ACP & $1 \mathrm{KLP}$ & $(46)$ & 1.8 & 2.5 \\
\hline E. coli holo-ACP & $1 \mathrm{ACP}$ & $(48)$ & & \\
\hline
\end{tabular}


Table 3

Comparison of the Acyl-binding Pockets of E. coli Butyryl-ACP, Spinach 10:0-ACP, and Spinach 18:0-ACP.

\begin{tabular}{|c|c|c|c|}
\hline \multicolumn{2}{|c|}{ Residues lining acvl-binding pocket } & \multicolumn{2}{c|}{ Atom contacts identified bv NOEs } \\
\hline $\begin{array}{c}\text { E. coli butyryl-ACP (from the X-ray } \\
\text { structure) (17) }\end{array}$ & $\begin{array}{c}\text { Spinach 10:0- and 18:0-ACP from CastP } \\
(\mathbf{3 9 , 4 0 )}\end{array}$ & Spinach 10:0-ACP & Spinach 18:0-ACP \\
\hline V7; V11 & V9 & & I6N \\
\hline F28 & F31 & F31CB; S32N & \\
\hline L42; V43; L46 & T41; I44; V45; L48 & T41N; I44CB; L48CB & L48N; E49N \\
\hline I54 & V56 & D 57N \\
\hline A59; M62 & A61; I64 & Q62N; I64N, CG1 & A61N; I64N \\
\hline A68; Y71; I72 & A70; V73; I74; L77 & A70CB; I74CA, CG1 & Q69N; V73N \\
\hline
\end{tabular}

